\title{
Alternative splicing during Arabidopsis flower development results in constitutive and stage-regulated isoforms
}

\author{
Haifeng Wang ${ }^{1,2+}$, Chenjiang You ${ }^{1+}$, Fang Chang ${ }^{1}$, Yingxiang Wang ${ }^{1}$, Lei Wang ${ }^{1}, \mathrm{Ji}^{\mathrm{Q}} \mathrm{i}^{1 *}$ and \\ Hong Ma ${ }^{1,2 *}$
}

' State Key Laboratory of Genetic Engineering and Institute of Plant Biology, Collaborative Innovation Center for Genetics and Development, School of Life Sciences, Fudan University, Shanghai, China

2 Institutes of Biomedical Sciences, Fudan University, Shanghai, China

\section{Edited by:}

Fangqing Zhao, Chinese Academy of Life Science, China

\section{Reviewed by:}

Suying Bao, The University of Hong

Kong, China

Cizhong Jiang, Tongji University,

China

*Correspondence:

Ji Qi, State Key Laboratory of Genetic Engineering and Institute of Plant Biology, Collaborative Innovation Center for Genetics and Development, School of Life Sciences, Fudan University, 220 Handan Rd., Shanghai 200433, China

e-mail: qij@fudan.edu.cn;

Hong Ma, State Key Laboratory of Genetic Engineering, Collaborative Innovation Center for Genetics and Development, School of Life Sciences, Institute of Plant Biology, Fudan University, 220 Handan Rd., Shanghai 200433, China and Institutes of Biomedical Sciences, Fudan University, 138 Yixueyuan Rd., Shanghai 200032, China e-mail: hongma@fudan.edu.cn

${ }^{t}$ These authors have contributed equally to this work.

\section{INTRODUCTION}

Alternative splicing (AS) produces multiple mRNAs from premRNAs with different splicing patterns, and has been detected widely in multicellular eukaryotes (Pan et al., 2008; Wang et al., 2008; Graveley et al., 2010). More than 95\% multi-exon genes have been estimated to have AS variants in human (Pan et al., 2008; Wang et al., 2008). AS plays important roles in regulating gene expression and increasing transcriptome diversity and proteome complexity. AS can produce transcripts that contain premature termination codons (PTCs), which are recognized by the non-sense mediated decay (NMD). NMD causes the degradation of such transcripts, thus reducing the abundance of splice variants. In the model plant Arabidopsis thaliana, approximately $50 \%$ of AS events are reported to produce transcripts with PTCs; for example, isoforms of the AFC2 and SOC1 genes have been found to contain PTCs (Filichkin et al., 2010; Marquez et al., 2012).
A number of studies suggested that AS can affect important biological processes in plants, including biotic/abiotic stress responses, photosynthesis, defense responses (Reddy, 2007), metabolic pathways (Gorlach et al., 1995), catabolic pathways (Kopriva et al., 1995), and flowering. Quesada et al. found that AS of FCA pre-mRNA prevented precocious flowering, by reducing its expression via cleavage and polyadenylation within the third intron and the production of a truncated non-functional transcript (Quesada et al., 2003). Recent studies estimated that 42-61\% of multi-exon genes in Arabidopsis experienced AS process (Filichkin et al., 2010; Marquez et al., 2012), potentially affecting a wide range of cellular processes.

Arabidopsis flower development has been an excellent model system to understand the molecular control of plant development (Bowman et al., 1989; Coen and Meyerowitz, 1991; Smyth, 2005). In particular, gene activities and regulation during Arabidopsis flower development have been investigated with transcriptome, 
often using microarray; however, standard microarray chips are not designed to detect alternatively spliced transcripts. The rapid advances in next generation sequencing technologies have facilitated numerous studies on AS profiles in many species by RNASeq, such as $H$. sapiens, D. melanogaster, S. cerevisiae, C. elegans, $A$. thaliana, and O. sativa (Nagalakshmi et al., 2008; Filichkin et al., 2010; Graveley et al., 2010; Zhang et al., 2010; Ramani et al., 2011; Loraine et al., 2013). However, in contrast to the studies of AS in animals, analysis of AS in the context of plant development is still at an early stage.

To characterize the complexity of AS in Arabidopsis flower development, we have used RNA-Seq to analyse genome-wide AS events in Arabidopsis at three different stages of flower development. This is the first time to perform such analyses with ultra-high throughput technology, providing an accurate and comprehensive evaluation of AS profile for Arabidopsis flower development. Using several AS prediction approaches and manual verification, we estimated that approximately $25 \%$ multi-exon genes underwent AS, less frequent than the rate for Arabidopsis multiple tissues (Filichkin et al., 2010; Marquez et al., 2012). Among the events we found, intron retention (IR) (approximately $50 \%$ ) was the most common type of AS compared to other types. The analysis further revealed that thousands of alternatively spliced transcripts were differentially expressed between different floral stages. We also identified many novel expressed regions considered unknown by the current annotations. Thus, our RNA-Seq data provide resources for comprehensive characterization of AS and gene expression in Arabidopsis flower development.

\section{MATERIALS AND METHODS \\ PLANT MATERIAL COLLECTION, RNA ISOLATION, AND SEOUENCING}

Plant materials representing three stages of flower development were collected from Arabidopsis thaliana [inflorescent meristem (IM), flower development stages from 1 to 9 , and flower development stage 12]. Considering the tiny structure of Arabidopsis IM and the difficulty it brings about to the sample collection, we used the IM from the aplcal mutant as a substitution, which proliferates inflorescence meristems and results in a cauliflower appearance (Bowman et al., 1993). Total RNA was isolated for each of the three stages and treated with TRIzol (Invitrogen) according to the manufacturer's instructions, then was subjected to 50 bp single-end sequencing on a SOLiD 3 platform (http:// www.appliedbiosystems.com). All SOLiD short reads have been submitted to NCBI Short Read Archive under accession number SRP035230.

\section{READ MAPPING AND TRANSCRIPTS ASSEMBLY}

A total of 420 million $50 \mathrm{bp}$ single-end reads were yielded for 5 samples from 3 stages. Reads from each sample were aligned to TAIR version 10 reference genome (www.arabidopsis.org) using Bowtie v0.12.7 (Langmead et al., 2009) and TopHat v1.3.2 (Trapnell et al., 2009) with a parameter "-C" for color space signal processing by SOLiD 3 platform. Intron sizes of predicted genes were limited to 50 and $5000 \mathrm{bp}$, to be consistent with those of annotated genes in the Arabidopsis genome (TAIR10), while the rest parameters were set as default. Cufflinks v0.93 (Trapnell et al., 2010) were then used to assemble the aligned reads, and to estimate the expression value of each assembled transcript.

\section{ALTERNATIVE SPLICING EVENTS IDENTIFICATION AND CLASSIFICATION}

To identify, in each stage, the AS events and their corresponding types, the ASTALAVISTA algorithm (Foissac and Sammeth, 2007) was used to predict AS events and determine the types of the events from the GTF files output by Cufflinks. The parameters of minimum and maximum intron length were fixed at 50 and $5000 \mathrm{bp}$ and the minimum reads covering novel identified transcripts should be no less than 15. Four AS types, namely intron retention (IR), exon skip (ES), alternative acceptor (AA), and alternative donor (AD), were extracted from the output of ASTALAVISTA for further analyses.

\section{GENE ONTOLOGY ENRICHMENT ANALYSIS}

To explore the functions of specifically expressed genes, we carried out GO enrichment analysis using the online AgriGO with Fisher's exact test (http://bioinfo.cau.edu.cn/agriGO/analysis. php). False discovery rate (FDR) correction was adopted with a threshold of 0.05 to reduce false positive prediction of enriched GO terms. A heatmap analysis for differentially expressed isoforms was implemented by using pheatmap (Pretty Heatmaps) function in an R package (pheatmap, version 0.74 ).

\section{Pfam DOMAIN ANNOTATION}

The protein domain annotation was downloaded from the Pfam database (http://pfam.sanger.ac.uk) (Punta et al., 2012). For known genes/transcripts, we obtained the Pfam annotation from TAIR10 (www.arabidopsis.org), and other novel predicted transcripts were searched against Pfam-A database with $e$-value cutoff as $1 \mathrm{e}-5$.

\section{REAL-TIME PCR VALIDATION OF GENE EXPRESSION AND ALTERNATIVE SPLICING EVENTS}

To verify the novel transcribed regions, we randomly selected 18 such regions to perform RT-PCR. ACT2 gene (AT3G18780) was used as a positive control, for which primers were designed on two neighboring exons. Total RNAs of Col-0 inflorescences were obtained by ZYMO RESEARCH ZR Plant RNA Miniprep ${ }^{\mathrm{TM}}$, and reverse transcription was carried out by TAKARA PrimeScript ${ }^{\mathrm{TM}}$ RT Master Mix. Forty cycles were run on ABI Veriti ${ }^{\circledR}$ 96-well Thermal Cycler with Tiangen Taq DNA Polymerase (ET101) and proper primers. The same cDNA library was also used for realtime PCR validation of genes with different expression values. The real-time PCR was performed with TAKARA SYBR ${ }^{\circledR}$ Premix Ex Taq $^{\mathrm{TM}}$ II (Tli RNaseH Plus) on ABI StepOnePlus ${ }^{\mathrm{TM}}$ Real Time system. Primers for these experiments are listed in Supplementary Table 7.

\section{RESULTS}

\section{TRANSCRIPTOME PROFILING AT THREE FLOWER DEVELOPMENT} STAGES OF A. thaliana

To investigate the transcriptomes of multiple stages in flower development, we isolated the total RNAs from three periods of flower development: IM stage, stage 1-9 (F1-9; early floral buds 
up to the time of meiosis) and stage 12 (F12; floral buds just before opening). Libraries were then constructed, and sequencing was performed on a SOLiD 3 platform, with one additional technique replicate for stages IM and F1-9. A total of 420 million high quality reads ( $50 \mathrm{bp}$, single-end) were obtained for the three stages of flower development, and $86 \%$ of them were mapped uniquely to the Arabidopsis reference genome (TAIR10) (Figure 1A). In addition, $82 \%$ of the mapped reads were aligned to proteincoding exonic regions, $9 \%$ to untranslated regions (UTR), 5\% to intergenic regions and $4 \%$ to intron regions (Figure 1B). According to the annotation information from TAIR10, expression of $80 \%$ of the annotated genes was detected in our samples; 95\% of the expressed genes were protein-coding while the rest included non-coding genes, micro RNA, transfer RNA, small nuclear RNA, and ribosome RNA (Figure 1C).

\section{THE EXPRESSION ESTIMATION OF GENES/TRANSCRIPTS ACROSS THREE FLOWER DEVELOPMENT STAGES}

The expression levels of genes and transcripts were estimated by applying Cufflinks (Trapnell et al., 2010) to short read alignment results for all three developmental stages. The comparison of the replicates of IM $\left(R^{2}=0.99\right)$ and F1-9 $\left(R^{2}=0.96\right)$ stages showed that the estimated levels of gene expression were highly consistent between the two replicates, especially for highly abundant genes (Figures 1D,E). The estimated gene expression was then used for further comparison among different flower development stages. The detailed statistics of read mapping were given in
Supplementary Tables 1, 2. Number of assembled transcripts and their corresponding genes were shown in Table 1. The majority of the aligned reads were further assembled by Cufflinks, resulting in 22,827 fully assembled transcripts (of the 25,245 annotated genes). FPKM (Fragments Per Kilobase of transcript per Million mapped reads) was then calculated to estimate the expression value for each gene and/or transcript separately.

To obtain an overview of the transcriptomes of flower development, we examined the distribution of gene expression values for each developmental stage. Each stage contained a group of genes with very low FPKM values, representing low expression or background. Taking a conservative approach, we used a cutoff of FPKM as 0.18 as a minimal expression value to avoid false positive estimation of gene expression, producing a unimodal distribution of genes expressed in each stage (Supplementary Figure 1). We detected 22,626 and 21,392 genes expressed at F12 and F1-9 stages, respectively (Figure 2A), much higher than the 14,833 and 14,460 genes previously reported for the same stages

Table 1 | Number of assembled genes and transcripts of the three flower development stages.

\begin{tabular}{lcc}
\hline Stages & Gene number & Transcript number \\
\hline IM & 21,181 & 30,608 \\
F1-9 & 22,137 & 29,759 \\
F12 & 22,827 & 33,791
\end{tabular}

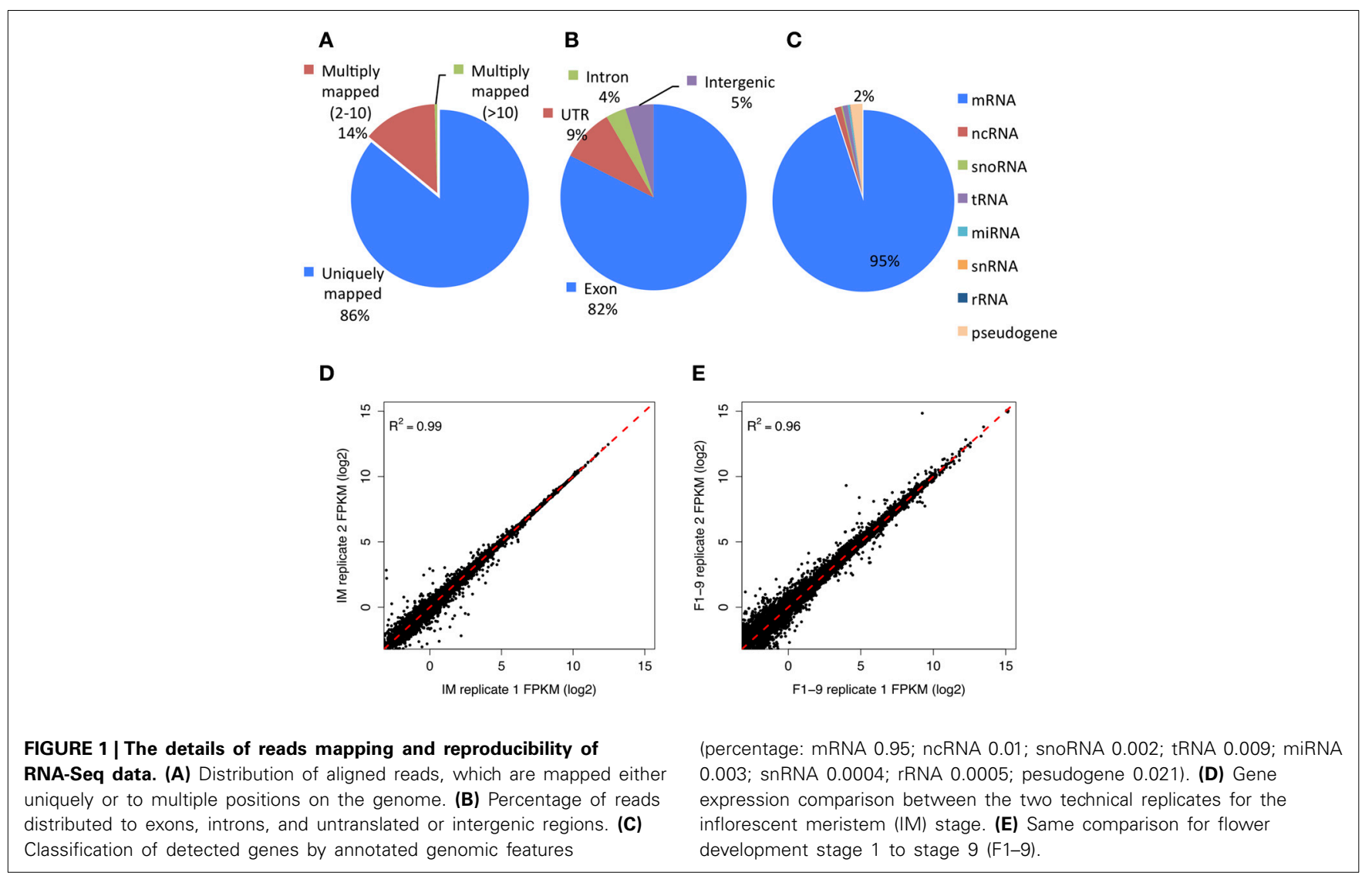




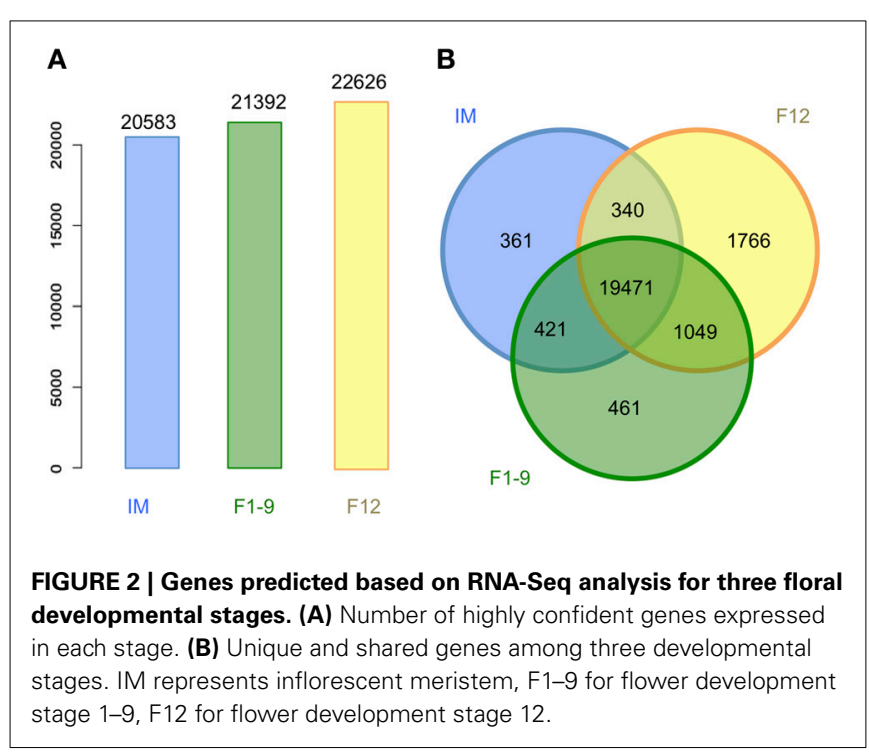

using microarray (Zhang et al., 2005). Compared with microarray technology, high throughput RNA-Seq technology could uncover the expression of many more genes.

We detected the expression of 20,583 genes at the IM stage, with a combined total of 23,860 genes (32,037 transcripts) expressed at the three stages of flower development; approximately $80 \%$ of these were detected at all three stages. We performed real-time PCR to validate the expression of 10 randomly selected genes, and the results were highly consistent with the estimates using RNA-Seq data (Supplementary Figure 2). In addition, 1049 genes were expressed at both the F1-9 and F12 stages but not in IM (Figure 2B), suggesting they are functionally important at relatively late stages of flower development. On the other hand, compared with the number of genes expressed only at IM (361) or at F1-9 (461), F12 had many more stage specific genes (1766), consistent with the fact that F12 contains more differentiated tissues and cells than the other two stages, particularly in the late stage of reproductive development, such as pollen and ovary development and so on.

\section{IDENTIFICATION OF ALTERNATIVE SPLICING EVENTS AND GENES}

To investigate the patterns of AS in flower development stages, we identified sequence reads supporting various types of AS events in our RNA-Seq data by using the ASTALAVISTA software (Foissac and Sammeth, 2007), which could detect the variations in the splicing structure and identify AS events by assigning each one an AS code, and has been applied to study AS in multiple Arabidopsis tissues (Marquez et al., 2012). In this study, we focused on four types of AS events: IR, AD, AA, and ES, by comparing RNA-Seq reads with annotated gene models. For example, we identified 4156 genes exhibiting AS among the 17,131 multi-exon genes of stage IM (Table 2). In total, we identified about $25.6 \%$ of TAIR10 annotated genes undergoing AS. Among the detected AS events, IR was the most abundant type (54.8 $\pm 0.98 \%)$, followed by $\mathrm{AA}(26.9 \pm 0.2 \%), \mathrm{AD}(13.1 \pm 0.5 \%)$, and $\mathrm{ES}(5.2 \pm 0.03 \%)$ (Table 3). These results were consistent with the recent findings in other studies (Filichkin et al., 2010; Zhang et al., 2010; Marquez
Table 2 | Estimation of alternatively spliced genes of the three stages.

\begin{tabular}{lcccc}
\hline & \multicolumn{3}{c}{ Flower stages (our study) } & \multirow{2}{*}{ TAIR10 } \\
\cline { 2 - 4 } & IM & F1-9 & F12 & \\
\hline Multi-exon genes & 17,131 & 18,048 & 18,326 & 22,352 \\
AS genes & 4156 & 3392 & 4466 & 5712 \\
\% of AS & $24.3 \%$ & $18.8 \%$ & $24.4 \%$ & $25.6 \%$ \\
\hline
\end{tabular}

et al., 2012). The observation that the percentage of IR events increased by approximately 14\% from that in a recent genomewide study in Arabidopsis using RNA-Seq (approximately 40\% IR events) suggested that IR was probably important for floral development.

\section{DIFFERENTIAL EXPRESSION OF ISOFORMS ACROSS FLORAL STAGES}

To investigate the possible role of alternatively spliced transcripts on flower development, expression levels of different isoforms of each gene were compared among the three stages. We found that 1716 alternatively spliced isoforms were differentially expressed between the three stages. These genes were further divided into four groups by hierarchical clustering algorithm by considering all the three stages simultaneously (Figure 3). Five hundred twenty-six isoforms (Cluster III in Figure 3) showed significantly higher expression levels at the IM stage than that at F1-9 and F12 stages, suggesting that these isoforms play roles in floral initiation. GO enrichment analyses were performed on this collection of isoforms and many functional categories were enriched including biological regulation, developmental processes, regulation of biological processes, and many biosynthetic processes. Among them, 19 genes were known to be important for flower development as demonstrated by genetic studies, such as CIB5, SFH3, AGL71, and MAF3 (Mo et al., 2007; Liu et al., 2008; Dorca-Fornell et al., 2011; Rosloski et al., 2013) (Supplementary Table 5). Furthermore, many genes encoding transcription factors were expressed at higher levels in IM than in the other two stages, including members of bHLH, MADS, ERF/AP2, NAC, bZIP, B-box, and other families, strongly suggesting that the alternatively spliced variants of transcription factors significantly increase the complexity of transcription regulation networks during flower development.

From four clusters of differentially expressed splicing isoforms, we also found that genes involved in response to stress/stimulus were preferentially expressed, with 48 isoforms in cluster I, and 57, 55, 74 isoforms in cluster II, III, IV, respectively. Taken together, those differentially expressed isoforms were likely important for transcription regulation and for response to internal or external stimulus during flower development.

\section{STAGE-SPECIFIC SPLICING ISOFORMS}

Comparing to the differentially expressed isoforms, stage-specific AS isoforms might be more important ( $\mathrm{Lv}$ et al., 2013). Before identifying alternatively spliced isoforms expressed specifically to each stage, we needed to find those genes exclusively expressed in each stage. The results showed that 13 genes out of 361 uniquely expressed genes were specifically alternatively spliced at stage IM, 
Table 3 | Summary of the four types of alternative splicing events of the three development stages.

\begin{tabular}{|c|c|c|c|c|c|c|}
\hline & \multicolumn{2}{|c|}{ IM } & \multicolumn{2}{|c|}{ F1-9 } & \multicolumn{2}{|c|}{ F12 } \\
\hline & Events & Genes & Events & Genes & Events & Genes \\
\hline IR & 2958 (54.8\%) & 2548 (52.9\%) & $2155(55.0 \%)$ & 1920 (50.4\%) & 3308 (56.6\%) & $2798(54.7 \%)$ \\
\hline ES & $284(5.2 \%)$ & $274(5.7 \%)$ & $243(5.3 \%)$ & $235(6.1 \%)$ & $272(4.7 \%)$ & 265 (5.2\%) \\
\hline AA & $1467(26.9 \%)$ & 1319 (27.5\%) & 1236 (27.1\%) & 1118 (29.3\%) & 1558 (26.7\%) & $1388(27.1 \%)$ \\
\hline$A D$ & $710(13.1 \%)$ & 667 (13.9\%) & $573(12.6 \%)$ & $540(14.2 \%)$ & 707 (12.1\%) & $667(13.0 \%)$ \\
\hline
\end{tabular}

$I R$, Intron retention; ES, Exon skiping; $A A$, Alternative acceptor; $A D$, Alternative donor.

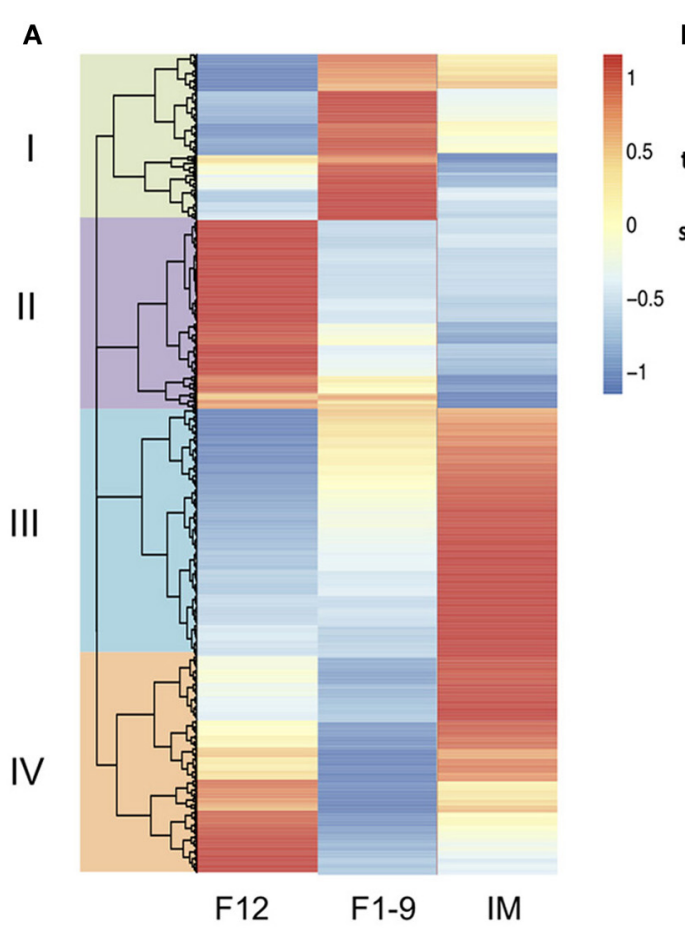

B

transcription factor activity

structural molecule activity

transcription regulator activity

reproductive process

regulation of biological process

metabolic process

biological regulation

response to stimulus

multicellular organismal process

developmental process

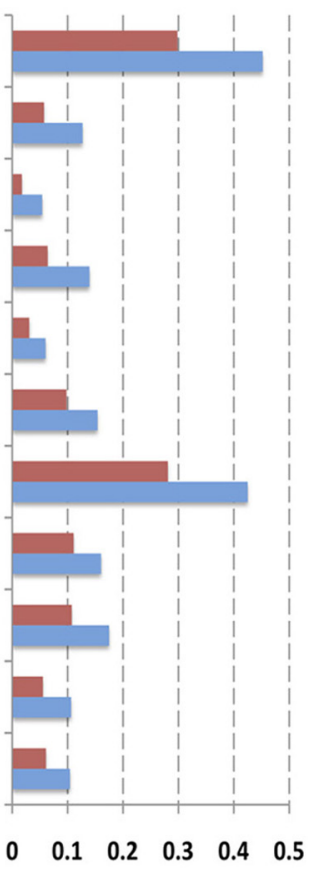

Percent of genes
FIGURE 3 | Hierarchical clustering and function enrichment analysis of 1716 differentially expressed isoforms among three stages. (A)

Differentially expressed isoforms were clustered into four clusters: 359 isoforms in cluster I (with expression values as F1-9 $>$ IM $>$ F12), 460

isoforms in cluster II (F12 $>$ F1-9 $>\mathrm{IM}), 401$ isoforms in cluster III (IM > F1-9
$>$ F12), and 526 isoforms in cluster IV (the others). Genes expressed higher than average are colored in red while lower in blue. (B) A list of enriched GO terms for genes in Cluster III. Red bars represent ratio of total annotated genes for each function, blue bars for differentially expressed genes within cluster III.
13 out of 461 genes and 66 out of 1766 genes were alternatively spliced at stage F1-9 and F12, respectively.

Among the 66 genes with specific splicing isoform(s) at F12 stage, AT2G30940, encoding a protein kinase, has two isoforms due to an $\mathrm{AD}$ event. As shown in Figure 4, the presence/absence of six nucleotides at the end of the fourth exon was observed in different isoforms, which were distinguished by 18 reads mapped to the exon-exon junction. Compared with the first transcript (AT2G30940.1), the second transcript (AT2G30940.2) encodes a protein with 2 extra amino acids. In addition, both transcripts were expressed at stage F12 only, with AT2G30940.1 more highly expressed than AT2G30940.2 (FPKM values: 1.9 vs. 0.01), suggesting that they provide different levels of function for Arabidopsis flower development. Similar phenomenon was observed for another gene (AT5G11400) encoding kinase and a gene (AT4G16162) coding for a Leucine-rich repeat (LRR) protein. Interestingly, many genes coding for protein kinases were found to be involved in the regulation of constitutive and AS in plants and animals through phosphorylation and interaction with serine/arginine-rich proteins (Birney et al., 2007), the regulation of several kinase genes by usage of alternative promoters and/or AS has been studied in animals (Duncan et al., 1995, 1997). Still, AS events of kinase genes and their effects on flower development of plants are little known, thus calling for further studies (Marquez et al., 2012).

Another important gene WRKY55 (AT2G40740) also expressed a splicing isoform specifically at stage F12. It is a member of the WRKY family, one of the largest families of plant-specific transcriptional regulators important for multiple plant processes. WRKY55 can bind specifically to the $\mathrm{W}$ box 

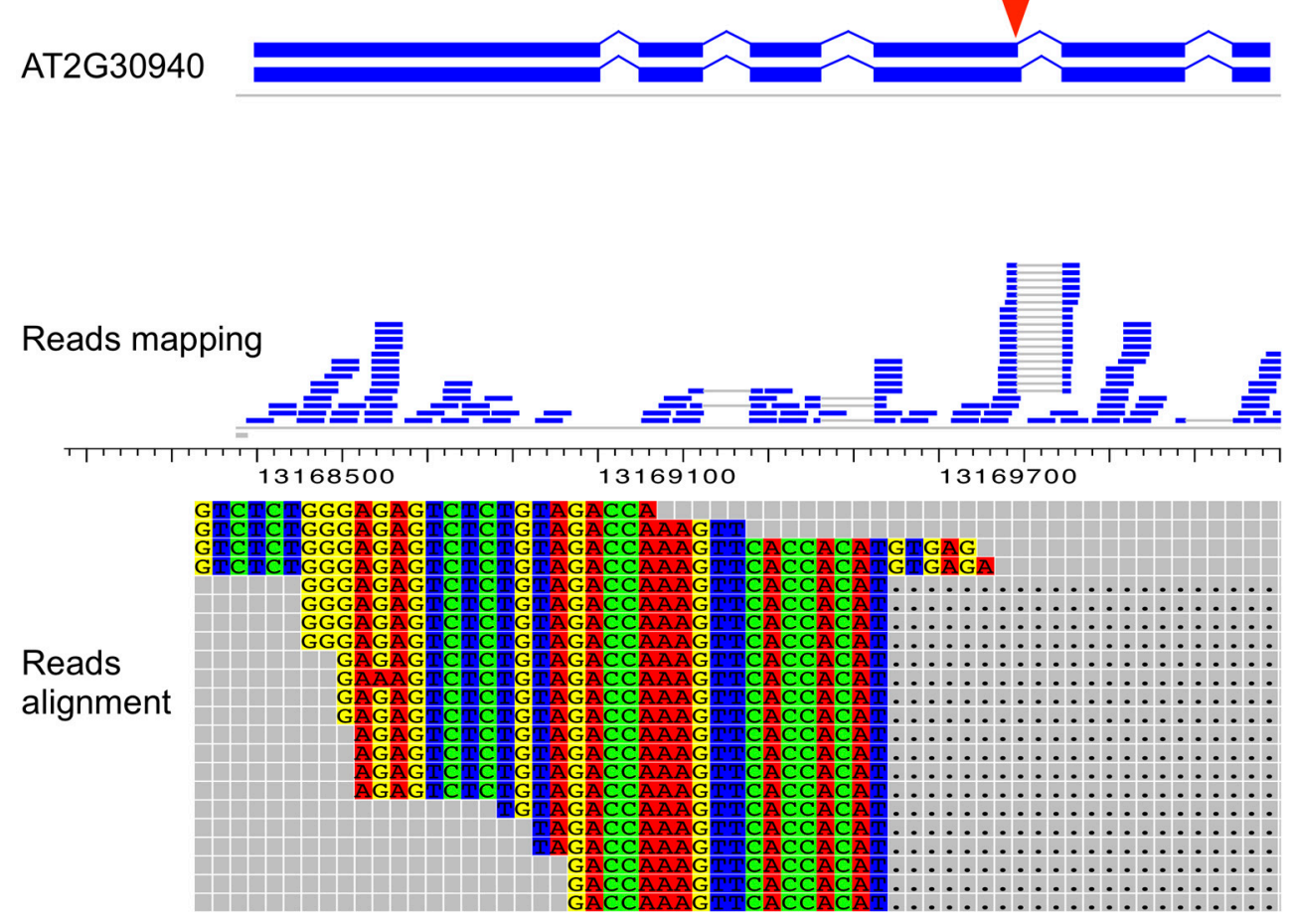

FIGURE 4 | An example of alternative spliced isoforms (of AT2G30940) expressed specifically at F12 stage. The fourth exons of the two isoforms end at different sites (pointed out in a red triangle) according to gene annotations in TAIR10, and this alternative donor event was further confirmed by PCR validation on flower tissues. Each bar (middle panel) represents a short read mapped to the reference genome. Two ends of a split read, indicting a cleavage of an intron, are connected by a gray line. One of the two transcripts, AT2G30940.1, is only expressed in F12, revealed both by alignment details of reads mapped to an exon-exon junction and by RT-PCR validation (relative ratio approximately 220.5, consistent with that of FPKM).
$\left(5^{\prime}-(\mathrm{T}) \mathrm{TGAC}[\mathrm{CT}]-3^{\prime}\right)$, a cis-acting element mediating elicitor responses. Previous studies proved that cis-acting elements were responsible for AS of many genes (Black, 2003; Higashide et al., 2004; Stamm et al., 2005), e.g., presenilin 2 (PS2) gene, which was abnormally alternatively spliced in a sample related to Alzheimer's disease (Higashide et al., 2004). For WRKY55, the ES event resulted in truncation of the WRKY domain, but the levels of expression were similar between these two isoforms ( 0.23 vs. 0.24 ; FPKM). Moreover, 36 other unknown functional genes with splicing isoforms specifically expressed at F12, providing particular materials for further genetic and genomic studies.

\section{IDENTIFICATION OF NOVEL TRANSCRIBED REGIONS FROM RNA-Seq DATA}

Compared with traditional technologies, RNA-Seq is able to identify novel transcribed regions without annotation from reads mapped to "intergenic" sequences. Considering the complex nature of Arabidopsis transcriptome, which contains many repetitive sequences from retro-transposons or recently duplicated genes, re-sequencing short reads from these variants could be wrongly mapped to non-allelic positions, making those regions marked as transcribed artificially. Thus only reads with mapping quality scores $\geq 20$ were considered uniquely mapped and were used in these analyses. We discovered 337 regions with average length of $457 \mathrm{bp}$, which were shorter than that of known genes $(2126 \mathrm{bp})$. An example of such putative novel genes with multiple exons is shown in Figure 5A. It is located on chromosome 1 between 913147 and 913739 with FPKM as 3.3 and was later verified by RT-PCR (Figure 5C). Each of the three exons of the gene was supported by five or more reads, while the two exon-exon junctions were also highly supported by multiple splitreads. We found that the expression distribution of these newly identified novel genes showed no significant difference from that of annotated genes ( $p$-value $>0.01$; Figure $5 B$ ), suggesting that they are truly transcribed regions with high confidence. However, a majority of them (263 of 337) had only a single exon and were approximately $500 \mathrm{bp}$ in length, with only a few longer than 1000 bp (Figure 5B). To verify those novel transcribed regions, we randomly selected 18 candidates for RT-PCR and 16 of them were confirmed, suggesting that the prediction of novel transcribed regions was highly accurate (Figure 5C). Furthermore, these novel identified genes lacked alternative splice variants. To determine whether they are functional will require further studies.

\section{DISCUSSION}

Previous studies used ESTs and microarrays to detect AS events, but these approaches are limited on both throughputs and sensitivities (Johnson et al., 2003; Iida et al., 2004). Recently developed RNA-Seq technologies with high throughput and reduced cost have greatly facilitated the comprehensive survey of gene expression and identification of AS events. In this study, we explored 


\section{A}

TAIR10

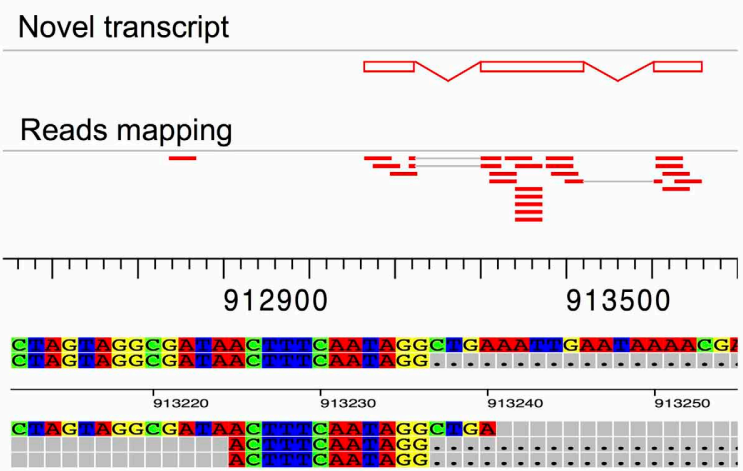

B

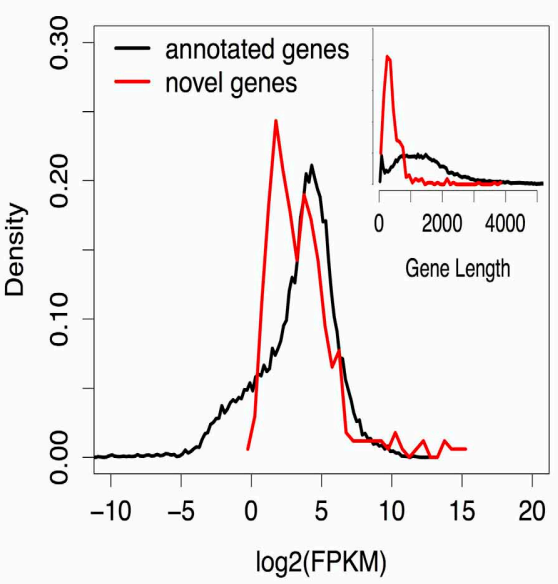

C

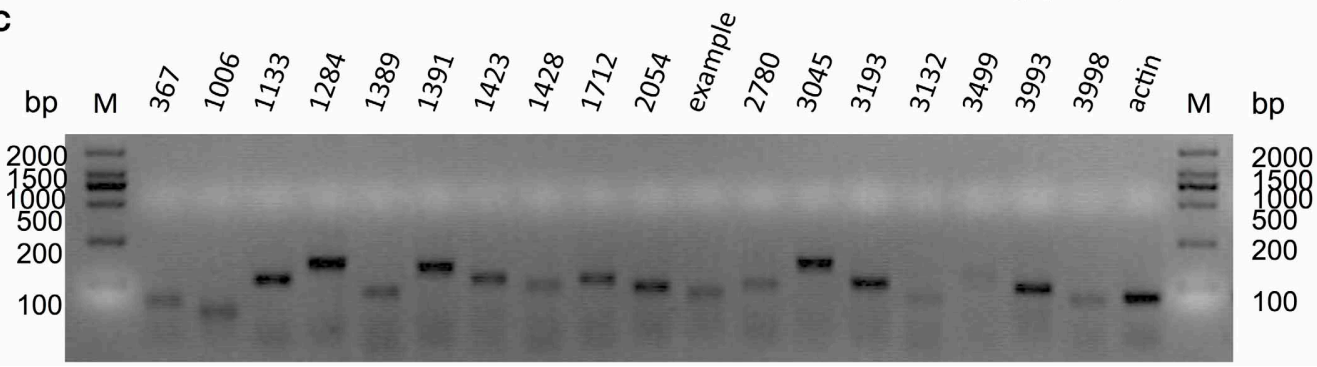

FIGURE 5 | Analysis of novel identified transcripts. (A) Display of a novel transcribed region identified by RNA-Seq analysis. It includes three exons, supported by short reads (in red bars). Detailed

alignment information (first exon-intron junction) is given in the lower panel. (B) Comparison of the expression values of novel transcripts and annotated genes. Comparison of gene lengths is given in the upper right panel. (C) RT-PCR validation of 18 randomly selected novel transcribed regions. the transcriptomes of three different stages of Arabidopsis flower development using RNA-Seq, and detected the expression of approximately 24,000 genes from the three stages. The reproducibility of our transcriptome data was supported by two technical replicates of the IM and F1-9 stages. Expression of many more genes was detected in this analysis than in previous studies using microarray at the same stages of flower development, indicating RNA-Seq is more sensitive (Zhang et al., 2005, 2010).

Furthermore, the ability to assemble short reads into transcripts allowed us to examine previously annotated AS events and identify novel alternatively spliced events. From the analysis of three developmental stages, we predicted approximately $25 \%$ of multi-exon genes undergo AS. IR was the most abundant type of AS in our analysis, accounting for more than $50 \%$ of total AS events, slightly more than that found in other tissues of Arabidopsis or in other plants (Zhang et al., 2010; Marquez et al., 2012). Our results are consistent with those in the recent studies, on the percentage of AS types, on Arabidopsis using RNA-Seq (Marquez et al., 2012). The greater frequency of AS from other recent works in Arabidopsis is probably due to the fact that they estimated the AS genes using multiple tissues and/or stages under different growth conditions (Barbazuk et al., 2008; Marquez et al., 2012). To exclude the influence of sequencing technology, we compared the AS events identified from our SOLiD sequencing with those from unpublished Illumina paired-end data in our laboratory. We found AS events based on SOLiD sequencing
(5845) were slightly fewer than those on Illumina platform (6132) and most of the predicted AS events were supported by both sequencing results, indicating these predictions were sequencing platform independent. The frequency of AS in plants are much lower than the estimates that up to $90 \%$ of human genes undergoing AS (Wang et al., 2008), suggesting that AS plays a less important role in gene regulation of plants than it does in animals, perhaps in part because plant genes can duplicate via several mechanisms, including whole-genome duplications (Blanc and Wolfe, 2004; Jiao et al., 2011).

Nevertheless, the AS isoforms identified here might be important for floral development since many of them are stageregulated. We identified 1716 alternatively spliced isoforms, including some for genes encoding transcription factor, were differentially expressed among the three stages. Therefore, these genes coding for transcription factor are regulated by both AS and differential expression, providing additional layers of mechanisms for regulating flower development. Other processes affected by differentially expressed AS isoforms include response to stress or stimulus, consistent with previous reports (Dinesh-Kumar and Baker, 2000; Staiger and Brown, 2013). We also detected AS of several protein kinase genes, as also found previously (Duncan et al., 1995, 1997); furthermore, AS was reported to be regulated by phosphorylation of SR proteins (Lareau et al., 2007), indicating a complex mutual regulatory interaction between AS and protein phosphorylation. Whether AS during flower development is 
also regulated by protein phosphorylation is an interesting question awaiting further studies. We also found several stage-specific spliced genes, including AT2G30940 that encoded a putative tyrosine kinase and produced AS transcripts by AD. The AS isoforms are similar in domain structure but have different expression levels. We found that AT2G30940 was only expressed at stage F12 comparing IM and F1-9 stage, suggesting that it is important for late flower development.

Furthermore, AS may alter the structure of translated proteins, such as retention/deletion and elongation/reduction of protein domains, thereby affecting function. According to the annotation of Pfam database (Punta et al., 2012), we found that 19.2\% of genes with annotated domains have length changes due to AS. Among these, genes encoding protein kinases were the most frequent, similar to previous observation (Punta et al., 2012); they have variable sizes from 61 to 377 aa that are associated with AS isoforms coding for proteins with truncated domains. Other domains with a wide range of sizes, e.g., K-box domain (from 42 to $100 \mathrm{aa}$ ), were also frequently affected by AS events (Supplementary Table 8).

Meanwhile, approximately $2.5 \%$ genes were identified to have their domains completely retained/deleted due to AS (see Supplementary Table 9 for the 10 most frequent types). For example, the WD40 domain defines a large family and is involved in several important biological processes including signal transduction, transcription regulation, and cell cycle control. We found that the WD40 domain is very frequently affected by AS, with various copy numbers of WD40 domains in different isoforms. Consistently, recent studies suggested that copy number changes of WD40 due to AS were found in many genes engaged in flowering transition (Ai et al., 2012) and other important regulatory network (Baurle and Dean, 2006). In addition, Zhou and his colleagues found that the WD40 domain of the Arabidopsis COP1 protein, which negatively regulates light-dependent development in the dark, could be deleted due to AS in a cop1 mutant (Zhou et al., 1998). This alternatively spliced isoform always occurs in mature seeds and in germinating seedlings and light independent (Zhou et al., 1998). Our findings that the number and size of domains are affected by AS suggest that different consequences of AS are important for the regulation of gene function and might have experienced selective pressure during evolution.

Due to the nature of computational algorithms for de novo gene prediction, only typical gene models could be wellannotated, while sequences generated by RNA-Seq provide an alternative way to annotate genes, facilitating the correction of exon-intron boundaries and de novo gene detection (Wang et al., 2009). In a recent study of the fruit fly Drosophila pseudoobscura, most of the 669 new genes uncovered by RNA-Seq data are located on unassembled contigs, indicating that the annotation of D. pseudoobscura was incomplete (Palmieri et al., 2012). While in plants, people began to utilize RNA-Seq results from multi-tissue to annotate genes ( $\mathrm{Li}$ et al., 2011). Our RNA-Seq analysis revealed 337 transcribed regions that were previously unknown and served to supplement the current Arabidopsis annotation. Some of these newly identified transcripts do not seem to encode proteins, suggesting that the RNA products might be the active form. Long non-coding RNAs have been identified from a number of organisms and are thought to play roles in regulating differentiation and stress response (Amor et al., 2009). Our results suggest that these transcripts could also play a role in regulating flower development.

In summary, our RNA-Seq analysis produced a comprehensive profile of AS during Arabidopsis flower development and provided valuable resources for investigating the regulation of gene expression and also facilitated further genomic and genetic studies.

\section{ACKNOWLEDGMENTS}

This work was supported by grant from the National Natural Science Foundation of China for Ji Qi (31371330) and for Hong Ma (31130006). We thank Zhihao Cheng, Genfeng Zhu, Hongxing Yang for technical support and deep discussion, and Yamao Chen for careful manuscript revise.

\section{SUPPLEMENTARY MATERIAL}

The Supplementary Material for this article can be found online at: http://www.frontiersin.org/journal/10.3389/fgene. 2014.00025/abstract

\section{REFERENCES}

Ai, X. Y., Lin, G., Sun, L. M., Hu, C. G., Guo, W. W., Deng, X. X., et al. (2012). A global view of gene activity at the flowering transition phase in precocious trifoliate orange and its wild-type [Poncirus trifoliata (L.) Raf.] by transcriptome and proteome analysis. Gene 510, 47-58. doi: 10.1016/j.gene.2012.07.090

Amor, B. B., Wirth, S., Merchan, F., Laporte, P., D’Äoaubenton-Carafa, Y., Hirsch, J., et al. (2009). Novel long non-protein coding RNAs involved in Arabidopsis differentiation and stress responses. Genome Res. 19, 57-69. doi: 10.1101/gr.080275.108

Barbazuk, W. B., Fu, Y., and McGinnis, K. M. (2008). Genome-wide analyses of alternative splicing in plants: opportunities and challenges. Genome Res. 18, 1381-1392. doi: 10.1101/Gr.053678.106

Baurle, I., and Dean, C. (2006). The timing of developmental transitions in plants. Cell 125, 655-664. doi: 10.1016/j.cell.2006.05.005

Birney, E., Stamatoyannopoulos, J. A., Dutta, A., Guigo, R., Gingeras, T. R., Margulies, E. H., et al. (2007). Identification and analysis of functional elements in $1 \%$ of the human genome by the ENCODE pilot project. Nature 447, 799-816. doi: 10.1038/nature05874

Black, D. L. (2003). Mechanisms of alternative pre-messenger RNA splicing. Annu. Rev. Biochem. 72, 291-336. doi: 10.1146/annurev.biochem.72.121801.161720

Blanc, G., and Wolfe, K. H. (2004). Widespread paleopolyploidy in model plant species inferred from age distributions of duplicate genes. Plant Cell 16, 1667-1678. doi: 10.1105/tpc.021345

Bowman, J. L., Alvarez, J., Weigel, D., Meyerowitz, E. M., and Smyth, D. R. (1993). Control of flower development in Arabidopsis thaliana by APETALA1 and interacting genes. Development. 119, 721-743.

Bowman, J. L., Smyth, D. R., and Meyerowitz, E. M. (1989). Genes directing flower development in Arabidopsis. Plant Cell 1, 37-52. doi: 10.1105/tpc.1.1.37

Coen, E. S., and Meyerowitz, E. M. (1991). The war of the whorls: genetic interactions controlling flower development. Nature 353, 31-37. doi: $10.1038 / 353031 \mathrm{a} 0$

Dinesh-Kumar, S. P., and Baker, B. J. (2000). Alternatively spliced N resistance gene transcripts: their possible role in tobacco mosaic virus resistance. Proc. Natl. Acad. Sci. U.S.A. 97, 1908-1913. doi: 10.1073/pnas.020367497

Dorca-Fornell, C., Gregis, V., Grandi, V., Coupland, G., Colombo, L., and Kater, M. M. (2011). The Arabidopsis SOC1-like genes AGL42, AGL71 and AGL72 promote flowering in the shoot apical and axillary meristems. Plant J. 67, 1006-1017. doi: 10.1111/j.1365-313X.2011.04653.x

Duncan, P. I., Howell, B. W., Marius, R. M., Drmanic, S., Douville, E. M., and Bell, J. C. (1995). Alternative splicing of STY, a nuclear dual specificity kinase. J. Biol. Chem. 270, 21524-21531. doi: 10.1074/jbc.270.37.21524 
Duncan, P. I., Stojdl, D. F., Marius, R. M., and Bell, J. C. (1997). In vivo regulation of alternative pre-mRNA splicing by the Clk1 protein kinase. Mol. Cell. Biol. 17, 5996-6001.

Filichkin, S. A., Priest, H. D., Givan, S. A., Shen, R. K., Bryant, D. W., Fox, S. E., et al. (2010). Genome-wide mapping of alternative splicing in Arabidopsis thaliana. Genome Res. 20, 45-58. doi: 10.1101/Gr.093302.109

Foissac, S., and Sammeth, M. (2007). ASTALAVISTA: dynamic and flexible analysis of alternative splicing events in custom gene datasets. Nucleic Acids Res. 35, W297-W299. doi: 10.1093/nar/gkm311

Gorlach, J., Raesecke, H. R., Abel, G., Wehrli, R., Amrhein, N., and Schmid, J. (1995). Organ-specific differences in the ratio of alternatively spliced chorismate synthase (LeCS2) transcripts in tomato. Plant J. 8, 451-456. doi: 10.1046/j.1365313X.1995.08030451.x

Graveley, B. R., Brooks, A. N., Carlson, J. W., Duff, M. O., Landolin, J. M., Yang, L., et al. (2010). The developmental transcriptome of Drosophila melanogaster. Nature 471, 473-479. doi: 10.1038/nature09715

Higashide, S., Morikawa, K., Okumura, M., Kondo, S., Ogata, M., Murakami, T., et al. (2004). Identification of regulatory cis-acting elements for alternative splicing of presenilin 2 exon 5 under hypoxic stress conditions. J. Neurochem. 91, 1191-1198. doi: 10.1111/j.1471-4159.2004.02798.x

Iida, K., Seki, M., Sakurai, T., Satou, M., Akiyama, K., Toyoda, T., et al. (2004). Genome-wide analysis of alternative pre-mRNA splicing in Arabidopsis thaliana based on full-length cDNA sequences. Nucleic Acids Res. 32, 5096-5103. doi: 10.1093/nar/gkh845

Jiao, Y., Wickett, N. J., Ayyampalayam, S., Chanderbali, A. S., Landherr, L., Ralph, P. E., et al. (2011). Ancestral polyploidy in seed plants and angiosperms. Nature 473, 97-100. doi: 10.1038/nature09916

Johnson, J. M., Castle, J., Garrett-Engele, P., Kan, Z., Loerch, P. M., Armour, C. D., et al. (2003). Genome-wide survey of human alternative pre-mRNA splicing with exon junction microarrays. Science 302, 2141-2144. doi: 10.1126/science. 1090100

Kopriva, S., Cossu, R., and Bauwe, H. (1995). Alternative splicing results in two different transcripts for $\mathrm{H}$-protein of the glycine cleavage system in the C4 species Flaveria trinervia. Plant J. 8, 435-441. doi: 10.1046/j.1365313X.1995.08030435.x

Langmead, B., Trapnell, C., Pop, M., and Salzberg, S. L. (2009). Ultrafast and memory-efficient alignment of short DNA sequences to the human genome. Genome Biol. 10:R25. doi: 10.1186/gb-2009-10-3-r25

Lareau, L. F., Inada, M., Green, R. E., Wengrod, J. C., and Brenner, S. E. (2007). Unproductive splicing of SR genes associated with highly conserved and ultraconserved DNA elements. Nature 446, 926-929. doi: 10.1038/Nature 05676

Li, Z., Zhang, Z., Yan, P., Huang, S., Fei, Z., and Lin, K. (2011). RNA-Seq improves annotation of protein-coding genes in the cucumber genome. BMC Genomics 12:540. doi: 10.1186/1471-2164-12-540

Liu, H., Yu, X., Li, K., Klejnot, J., Yang, H., Lisiero, D., et al. (2008). Photoexcited CRY2 interacts with CIB1 to regulate transcription and floral initiation in Arabidopsis. Science 322, 1535-1539. doi: 10.1126/science.1163927

Loraine, A. E., McCormick, S., Estrada, A., Patel, K., and Qin, P. (2013). RNA-seq of Arabidopsis pollen uncovers novel transcription and alternative splicing. Plant Physiol. 162, 1092-1109. doi: 10.1104/pp.112.211441

Lv, Y., Zuo, Z., and Xu, X. (2013). Global detection and identification of developmental stage specific transcripts in mouse brain using subtractive crossscreening algorithm. Genomics 102, 229-236. doi: 10.1016/j.ygeno.2013.05.001

Marquez, Y., Brown, J. W., Simpson, C., Barta, A., and Kalyna, M. (2012). Transcriptome survey reveals increased complexity of the alternative splicing landscape in Arabidopsis. Genome Res. 22, 1184-1195. doi: 10.1101/gr.134106.111

Mo, P., Zhu, Y., Liu, X., Zhang, A., Yan, C., and Wang, D. (2007). Identification of two phosphatidylinositol/phosphatidylcholine transfer protein genes that are predominately transcribed in the flowers of Arabidopsis thaliana. J. Plant Physiol. 164, 478-486. doi: 10.1016/j.jplph.2006.03.014

Nagalakshmi, U., Wang, Z., Waern, K., Shou, C., Raha, D., Gerstein, M., et al. (2008). The transcriptional landscape of the yeast genome defined by RNA sequencing. Science 320, 1344-1349. doi: 10.1126/science.1158441

Palmieri, N., Nolte, V., Suvorov, A., Kosiol, C., and Schlotterer, C. (2012). Evaluation of different reference based annotation strategies using RNA-Seq - a case study in Drososphila pseudoobscura. PLoS ONE 7:e46415. doi: 10.1371/journal.pone.0046415
Pan, Q., Shai, O., Lee, L. J., Frey, J., and Blencowe, B. J. (2008). Deep surveying of alternative splicing complexity in the human transcriptome by high-throughput sequencing. Nat. Genet. 40, 1413-1415. doi: 10.1038/Ng.259

Punta, M., Coggill, P. C., Eberhardt, R. Y., Mistry, J., Tate, J., Boursnell, C., et al. (2012). The Pfam protein families database. Nucleic Acids Res. 40, D290-D301. doi: 10.1093/nar/gkt1223

Quesada, V., Macknight, R., Dean, C., and Simpson, G. G. (2003). Autoregulation of FCA pre-mRNA processing controls Arabidopsis flowering time. EMBO J. 22, 3142-3152. doi: 10.1093/emboj/cdg305

Ramani, A. K., Calarco, J. A., Pan, Q., Mavandadi, S., Wang, Y., Nelson, A. C., et al. (2011). Genome-wide analysis of alternative splicing in Caenorhabditis elegans. Genome Res. 21, 342-348. doi: 10.1101/gr.114645.110

Reddy, A. S. (2007). Alternative splicing of pre-messenger RNAs in plants in the genomic era. Annu. Rev. Plant Biol. 58, 267-294. doi: 10.1146/annurev.arplant. 58.032806.103754

Rosloski, S. M., Singh, A., Jali, S. S., Balasubramanian, S., Weigel, D., and Grbic, V. (2013). Functional analysis of splice variant expression of MADS AFFECTING FLOWERING 2 of Arabidopsis thaliana. Plant Mol. Biol. 81, 57-69. doi: $10.1007 /$ s11103-012-9982-2

Smyth, D. R. (2005). Morphogenesis of flowers-our evolving view. Plant Cell 17, 330-341. doi: 10.1105/tpc.104.030353

Staiger, D., and Brown, J. W. (2013). Alternative splicing at the intersection of biological timing, development, and stress responses. Plant Cell 25, 3640-3656. doi: 10.1105/tpc.113.113803

Stamm, S., Ben-Ari, S., Rafalska, I., Tang, Y., Zhang, Z., Toiber, D., et al. (2005). Function of alternative splicing. Gene 344, 1-20. doi: 10.1016/j.gene. 2004.10.022

Trapnell, C., Pachter, L., and Salzberg, S. L. (2009). TopHat: discovering splice junctions with RNA-Seq. Bioinformatics 25, 1105-1111. doi: 10.1093/Bioinformatics/Btp120

Trapnell, C., Williams, B. A., Pertea, G., Mortazavi, A., Kwan, G., Van Baren, M. J., et al. (2010). Transcript assembly and quantification by RNA-Seq reveals unannotated transcripts and isoform switching during cell differentiation. Nat. Biotechnol. 28, 511-515. doi: 10.1038/nbt.1621

Wang, E. T., Sandberg, R., Luo, S. J., Khrebtukova, I., Zhang, L., Mayr, C., et al. (2008). Alternative isoform regulation in human tissue transcriptomes. Nature 456, 470-476. doi: 10.1038/Nature07509

Wang, Z., Gerstein, M., and Snyder, M. (2009). RNA-Seq: a revolutionary tool for transcriptomics. Nat. Rev. Genet. 10, 57-63. doi: 10.1038/nrg2484

Zhang, G., Guo, G., Hu, X., Zhang, Y., Li, Q., Li, R., et al. (2010). Deep RNA sequencing at single base-pair resolution reveals high complexity of the rice transcriptome. Genome Res. 20, 646-654. doi: 10.1101/gr. 100677.109

Zhang, X. H., Feng, B. M., Zhang, Q., Zhang, D. Y., Altman, N., and Ma, H. (2005). Genome-wide expression profiling and identification of gene activities during early flower development in Arabidopsis. Plant Mol. Biol. 58, 401-419. doi: 10.1007/S11103-005-5434-6

Zhou, D. X., Kim, Y. J., Li, Y. F., Carol, P., and Mache, R. (1998). COP1b, an isoform of COP1 generated by alternative splicing, has a negative effect on COP1 function in regulating light-dependent seedling development in Arabidopsis. Mol. Gen. Genet. 257, 387-391. doi: 10.1007/s004380050662

Conflict of Interest Statement: The authors declare that the research was conducted in the absence of any commercial or financial relationships that could be construed as a potential conflict of interest.

Received: 16 December 2013; accepted: 24 January 2014; published online: 12 February 2014.

Citation: Wang H, You C, Chang F, Wang Y, Wang L, Qi J and Ma H (2014) Alternative splicing during Arabidopsis flower development results in constitutive and stage-regulated isoforms. Front. Genet. 5:25. doi: 10.3389/fgene.2014.00025

This article was submitted to Bioinformatics and Computational Biology, a section of the journal Frontiers in Genetics.

Copyright (c) 2014 Wang, You, Chang, Wang, Wang, Qi and Ma. This is an openaccess article distributed under the terms of the Creative Commons Attribution License (CC BY). The use, distribution or reproduction in other forums is permitted, provided the original author(s) or licensor are credited and that the original publication in this journal is cited, in accordance with accepted academic practice. No use, distribution or reproduction is permitted which does not comply with these terms. 